\title{
Dimensional Crossover of Dilute Neon inside Infinitely Long Single-Walled Carbon Nanotubes Viewed from Specific Heats
}

\author{
Zhan-chun $\mathrm{Tu}^{1,2, *}$ and Zhong-can Ou-Yang ${ }^{1,3}$ \\ ${ }^{1}$ Institute of Theoretical Physics, The Chinese Academy of Sciences, \\ P.O.Box 2735 Beijing 100080, China \\ ${ }^{2}$ Graduate School, The Chinese Academy of Sciences, Beijing, China \\ ${ }^{3}$ Center for Advanced Study, Tsinghua University, Beijing 100084, China
}

\begin{abstract}
A simple formula for coordinates of carbon atoms in a unit cell of a single-walled nanotube (SWNT) is presented and the potential of neon (Ne) inside an infinitely long SWNT is analytically derived under the assumption of pair-wise Lennard-Jones potential between Ne and carbon atoms. Specific heats of dilute Ne inside infinitely long $(5,5),(10,10),(15,15)$ and $(20,20)$ SWNT's are calculated at different temperatures. It is found that Ne inside four kinds of nanotubes exhibits 3-dimensional (3D) gas behavior at high temperature but different behaviors at low temperature: Ne inside $(5,5)$ nanotube behaves as $1 \mathrm{D}$ gas but inside $(10,10),(15,15)$, and $(20,20)$ nanotubes behaves as 2D gas. Furthermore, at ultra low temperature, Ne inside $(5,5)$ nanotube still displays 1D behavior but inside $(10,10),(15,15)$, and $(20,20)$ nanotubes behaves as lattice gas.
\end{abstract}

PACS numbers: 61.46.+w, 82.60.Fa 
Since the discovery of carbon nanotubes, $\frac{1}{\stackrel{1}{*}}$ the peculiar electronic and mechanical properties of these structures have attracted much attention.2.3.4 Experiments have also revealed that carbon nanotubes can be used to store hydrogen ${ }^{\frac{5}{-}}$ and other gases. ${ }^{6}$ Many physicists expect gases in nanotubes or nanotube bundles to display 1-dimensional (1D) behavior as a consequence of the remarkable aspect ratio of the length of tubes to their radius. Cole and his co-workers ${ }^{7}$ and other researchers ${ }^{8}$ have theoretically studied properties of gases in nanotubes or nanotube bundles. One of the most fantastic properties they found is specific heat of dilute gas inside single-walled carbon nanotubes (SWNT's) as a functions of temperature: With temperature increasing it shows the thermal behavior changing from $1 \mathrm{D}$ to 2D, to 3D. However, there is still a question: Do $1 \mathrm{D}$ and $2 \mathrm{D}$ behaviors always exist for dilute gas inside SWNT's if the temperature is low enough?

A series of skillful experiments to measure specific heat of gas in carbon nanotube bundles were done by Lasjaunias et al. $\frac{9}{\cdot}$ In their experiments, He atoms are adsorbed within the interstitials or external grooves and surfaces of the bundles, and exhibit 1D behavior at low temperature. Their experiments suggest that the specific heat can reflect the dimensional information well.

Here we firstly present a simple formula for coordinates of carbon atoms in a unit cell of a SWNT and derive an analytical expression of the potential of neon ( $\mathrm{Ne}$ ) inside an infinitely long SWNT under the assumption of pair-wise Lennard-Jones potential between Ne and carbon atoms. And then, we calculate specific heats of dilute Ne inside $(5,5),(10,10),(15$, 15) and $(20,20)$ SWNT's and find that Ne inside four kinds of nanotubes exhibits 3D gas behavior at high temperature but different behaviors at low temperature: Ne inside $(5,5)$ nanotube behaves as 1D gas but inside $(10,10),(15,15)$, and $(20,20)$ nanotubes behaves as 2D gas. Furthermore, at ultra low temperature, Ne inside $(5,5)$ nanotube still displays $1 \mathrm{D}$ behavior but inside $(10,10),(15,15)$, and $(20,20)$ nanotubes behaves as lattice gas. Ne inside $(5,5)$ nanotube does not display 2D behavior and inside (10, 10), (15, 15), and (20, 20) nanotubes does not display 1D behavior. Thus Ne inside nanotubes does not always display 1D behavior in spite of the remarkable aspect ratio of the length of tubes to their radius. Besides, Ne inside a definite nanotube may display neither 1D nor 2D behaviors at different temperatures.

A SWNT without two caps can be constructed by wrapping up a single sheet of graphite such that two equivalent sites of hexagonal lattice coincide ${ }^{10}$ To describe the SWNT, some 
characteristic vectors require introducing. As shown in Fig 1 the chiral vector $\mathbf{C}_{h}$, which defines the relative location of two sites, is specified by a pair of integers $\left(n_{1}, n_{2}\right)$ which is called the index of the SWNT and relates $\mathbf{C}_{h}$ to two unit vectors $\mathbf{a}_{1}$ and $\mathbf{a}_{2}$ of graphite $\left(\mathbf{C}_{h}=n_{1} \mathbf{a}_{1}+n_{2} \mathbf{a}_{2}\right)$. The translational vector $\mathbf{T}$ corresponds to the first lattice point of $2 \mathrm{D}$ graphitic sheet through which the line normal to the chiral vector $\mathbf{C}_{h}$ passes. The unit cell of the SWNT is the rectangle defined by vectors $\mathbf{C}_{h}$ and $\mathbf{T}$, while vectors $\mathbf{a}_{1}$ and $\mathbf{a}_{2}$ define the area of the unit cell of 2D graphite. The number $N$ of hexagons per unit cell of SWNT is obtained as a function of $n_{1}$ and $n_{2}$ as $N=2\left(n_{1}^{2}+n_{2}^{2}+n_{1} n_{2}\right) / d_{R}$, where $d_{R}$ is the greatest common divisor of $\left(2 n_{2}+n_{1}\right)$ and $\left(2 n_{1}+n_{2}\right)$. There are $2 N$ carbon atoms in each unit cell of SWNT because every hexagon contains two atoms. To denote the $2 N$ atoms, we use a symmetry vector $\mathbf{R}$ to generate coordinates of carbon atoms in the nanotube and define it as the site vector having the smallest component in the direction of $\mathbf{C}_{h}$. From a geometric standpoint, vector $\mathbf{R}$ consists of a rotation around the nanotube axis by an angle $\Psi=2 \pi / N$ combined with a translation $\tau$ in the direction of $\mathbf{T}$; therefore, $\mathbf{R}$ can be denoted by $\mathbf{R}=(\Psi \mid \tau)$. Using the symmetry vector $\mathbf{R}$, we can divide the $2 N$ carbon atoms in the unit cell of SWNT into two groups: one includes $N$ atoms whose site vectors satisfy

$$
\mathbf{A}_{l}=l \mathbf{R}-\left[l \mathbf{R} \cdot \mathbf{T} / \mathbf{T}^{2}\right] \mathbf{T} \quad(l=0,1,2, \cdots, N-1),
$$

another includes the remainder $N$ atoms whose site vectors satisfy

$$
\begin{aligned}
\mathbf{B}_{l} & =l \mathbf{R}+\mathbf{B}_{0}-\left[\left(l \mathbf{R}+\mathbf{B}_{0}\right) \cdot \mathbf{T} / \mathbf{T}^{2}\right] \mathbf{T} \\
& -\left[\left(l \mathbf{R}+\mathbf{B}_{0}\right) \cdot \mathbf{C}_{h} / \mathbf{C}_{h}^{2}\right] \mathbf{C}_{h} \quad(l=0,1,2, \cdots, N-1),
\end{aligned}
$$

where $\mathbf{B}_{0}$ represents one of the nearest neighbor atoms to $\mathbf{A}_{0}$. In and only in above two equations, [...] denotes the Gaussian function, e.g., [5.3] $=5$.

To obtain the potential of Ne inside the nanotube, we firstly consider another simple system shown in Fig. 2. Many carbon atoms distributed regularly in a line form an infinite atom chain and a Ne atom $\mathrm{Q}$ is out of the chain. The interval between neighbor atoms in the chain is $T$, and the site of atom $\mathrm{Q}$ relative to atom 0 can be represented by two numbers $c_{1}$ and $c_{2}$. We take the Lennard-Jones potential $U\left(R_{j}\right)=4 \epsilon\left[\left(\sigma / R_{j}\right)^{12}-\left(\sigma / R_{j}\right)^{6}\right]$ between atom $\mathrm{Q}$ and atom $j$ in the chain, where $R_{j}$ is the distance between $\mathrm{Q}$ and atom $j$, and $\epsilon=\sqrt{\epsilon_{c} \epsilon_{n e}}$, $\sigma=\left(\sigma_{c}+\sigma_{n e}\right) / 2$ with $\epsilon_{n e}=35.6 \mathrm{~K}, \sigma_{n e}=2.75 \AA, \epsilon_{c}=28 \mathrm{~K}$ and $\sigma_{c}=3.4 \AA .7 .11$ We express 
the potential between atom $\mathrm{Q}$ and the chain as

$$
U_{Q C}=4 \epsilon\left[\sigma^{12} U_{6}\left(c_{1}, c_{2}\right)-\sigma^{6} U_{3}\left(c_{1}, c_{2}\right)\right]
$$

where $U_{k}\left(c_{1}, c_{2}\right)=\sum_{n=-\infty}^{\infty} \frac{1}{\left[\left(c_{1}+n T\right)^{2}+c_{2}^{2}\right]^{k}} \quad(k=1,2, \cdots)$ which can be calculated through the following recursion: $\frac{12}{12}$

$$
\begin{aligned}
& U_{1}\left(c_{1}, c_{2}\right)=\frac{\pi \sinh \left(2 \pi c_{2} / T\right)}{c_{2} T\left[\cosh \left(2 \pi c_{2} / T\right)-\cos \left(2 \pi c_{1} / T\right)\right]}, \\
& U_{k+1}\left(c_{1}, c_{2}\right)=-1 /\left(2 k c_{2}\right) \partial U_{k} / \partial c_{2} .
\end{aligned}
$$

The $(20,20)$ tube, for example, with infinite length can be regarded as $2 N=80$ chains. Thus the potential of any point $\mathrm{Q}$ inside the tube can be calculated as

$$
U(r, \theta, z)=\sum_{i=1}^{2 N} U_{Q C}
$$

where $(r, \theta, z)$ is coordinates of $\mathrm{Q}$ in the cylindrical coordinate system whose $z$-axis is the tube axis, $r$ the distance between $\mathrm{Q}$ and $z$-axis, and $\theta$ the angle rotating around $z$-axis from an axis which is vertical to $z$-axis and passes through atom $A_{0}$ on the tube to the plane that contains $\mathrm{Q}$ and $z$-axis. As an approximation, we neglect the potential varying with $z$ and $\theta$ because we find that it is much smaller than the potential varying with $r$ through calculations, and fit the potential with $U(r)=4 \varepsilon\left[\left(\frac{\tilde{\sigma}}{\rho-r}\right)^{10}-\left(\frac{\tilde{\sigma}}{\rho-r}\right)^{4}\right]$, where $\rho=13.56 \AA$ is the radius of the tube, $\varepsilon=390 \mathrm{~K}$, and $\tilde{\sigma}=2.63 \AA$ (see also Fig 3 ). Moreover, we simplify it as

$$
U(r)=\left\{\begin{array}{l}
4 \varepsilon\left[\left(\frac{\tilde{\sigma}}{\rho-r}\right)^{10}-\left(\frac{\tilde{\sigma}}{\rho-r}\right)^{4}\right], \quad r<\rho-\tilde{\sigma} \\
\infty, \quad r>\rho-\tilde{\sigma} .
\end{array}\right.
$$

Because we consider the dilute Ne, we can neglect the interaction between Ne atoms and write the single particle Schrödinger equation ${ }^{13}$ as $H \psi=E \psi$, where $H=-\frac{\hbar^{2}}{2 \mu} \nabla^{2}+U(r)$ and $\psi=\phi e^{i(m \theta+\kappa z)}$. From them we arrive at

$$
\begin{aligned}
& E=\frac{\hbar^{2} \kappa^{2}}{2 \mu}+E_{m} \quad(\kappa \in \mathbb{R}, m=0, \pm 1, \pm 2, \cdots), \\
& H(r) \phi=E_{m} \phi, \\
& H(r)=-\frac{\hbar^{2}}{2 \mu}\left(\frac{d^{2}}{d r^{2}}+\frac{1}{r} \frac{d}{d r}-\frac{m^{2}}{r^{2}}\right)+U(r) .
\end{aligned}
$$

Setting $r=(\rho-\tilde{\sigma}) \xi, \varepsilon_{0}=\frac{\hbar^{2}}{2 \mu \rho^{2}}$ and $\eta=\tilde{\sigma} / \rho$, Eqs.(6) and (17) are transformed into

$$
u(\xi)=\left\{\begin{array}{l}
4 \varepsilon\left[\left(\frac{\eta}{1-(1-\eta) \xi}\right)^{10}-\left(\frac{\eta}{1-(1-\eta) \xi}\right)^{4}\right], \quad \xi<1 \\
\infty, \quad \xi>1
\end{array}\right.
$$


and

$$
\begin{aligned}
& \mathcal{H} \varphi(\xi)=E_{m} \varphi(\xi), \\
& \mathcal{H}=-\frac{\varepsilon_{0}}{(1-\eta)^{2}}\left(\frac{d^{2}}{d \xi^{2}}+\frac{1}{\xi} \frac{d}{d \xi}-\frac{m^{2}}{\xi^{2}}\right)+u(\xi) .
\end{aligned}
$$

If we let $|\varphi\rangle=\sum_{n} a_{n}\left|\chi_{n}\right\rangle$, we will obtain the secular equation

$$
\operatorname{det}\left(\mathcal{H}_{j n}-E_{m} \mathcal{S}_{j n}\right)=0
$$

where $\mathcal{H}_{j n}=\int_{0}^{1} \chi_{j} \mathcal{H}(\xi) \chi_{n} \xi d \xi$, and $\mathcal{S}_{j n}=\int_{0}^{1} \chi_{j} \chi_{n} \xi d \xi$. If we let $\chi_{n}=J_{|m|}\left(\nu_{n} \xi\right)$, where $J_{|m|}(\xi)$ is the m-th order Bessel function of the first class and $\nu_{n}$ is the $n$-th zero point of Bessel function, ${ }^{14}$ we can calculated $E_{m n},(m=0, \pm 1, \pm 2, \cdots ; n=1,2,3, \ldots)$ from Eq.(10).

If there are $\mathcal{N}$ Ne atoms inside the tube, we have the free energy $\mathcal{F}=-\mathcal{N} \mathcal{T} \ln \mathcal{Z}$, where $\mathcal{T}$ is the temperature, $\mathcal{Z}=\sum_{m n} e^{-E_{m n} / \mathcal{T}} \int_{-\infty}^{\infty} e^{-\frac{\hbar^{2} \kappa^{2}}{2 \mu \mathcal{T}}} d \kappa$ and the Boltzmann factor is set to $1 .^{15}$ We can easily obtain the specific heat per atom is

$$
c_{v}=-\frac{\mathcal{T} \partial^{2} \mathcal{F}}{\mathcal{N} \partial \mathcal{T}^{2}}=\frac{1}{2}+\frac{\left\langle E^{2}\right\rangle-\langle E\rangle^{2}}{\mathcal{T}^{2}},
$$

where $\langle E\rangle=\frac{\sum_{m n} E_{m n} e^{-E_{m n} / \mathcal{T}}}{\sum_{m n} e^{-E_{m n} / \mathcal{T}}}$ and $\left\langle E^{2}\right\rangle=\frac{\sum_{m n} E_{m n}^{2} e^{-E_{m n} / \mathcal{T}}}{\sum_{m n} e^{-E_{m n} / \mathcal{T}}}$.

In Fig 4 , the symbols " $\nabla$ " reflect $c_{v}$ varying with temperature $\mathcal{T}$, which implies Ne inside $(20,20)$ tube behaves as 3D gas at high temperature (specific heat approaches to $3 / 2)$ and $2 \mathrm{D}$ gas at low temperature (specific heat is 1 ). Therefore, we can naturally assume that all atoms in the valley of potential $U(r)$ at low temperature, i.e. lie on the shell $S^{*}$ with radius $\varrho=\rho\left[1-(5 / 2)^{1 / 6} \eta\right]$.

Now we consider the thermal property of Ne inside the nanotube at low temperature more carefully. From Eqs.(3) -(5) we can easily calculate the potential $U_{s}(v, z)$ on $S^{*}$, where $v=\varrho \theta$. The Hamiltonian of single particle can be expressed as $H^{\prime}=-\frac{\hbar^{2}}{2 \mu}\left(\frac{\partial^{2}}{\partial v^{2}}+\frac{\partial^{2}}{\partial z^{2}}\right)+U_{s}(v, z)$.

In fact, $U_{s}$ has periodic structure. If we denote $\boldsymbol{\alpha}_{1}=(2 \pi \varrho / N, \tau), \boldsymbol{\alpha}_{2}=(0, T)$ and $\gamma_{l}=l_{1} \boldsymbol{\alpha}_{1}+l_{2} \boldsymbol{\alpha}_{2}$, we know $U_{s}\left(\mathbf{r}+\gamma_{l}\right)=U_{s}(\mathbf{r})$, where $l_{1}, l_{2} \in \mathbb{Z}$ and $\mathbf{r}=(v, z)$. On the one hand, we have the Bloch's theorem: ${ }^{16}$

$$
\begin{aligned}
& H^{\prime} \Phi(\boldsymbol{\kappa}, \mathbf{r})=E_{\boldsymbol{\kappa}} \Phi(\boldsymbol{\kappa}, \mathbf{r}) \\
& \Phi\left(\boldsymbol{\kappa}, \mathbf{r}+\boldsymbol{\gamma}_{l}\right)=e^{i \boldsymbol{\kappa} \cdot \boldsymbol{\gamma}_{l}} \Phi(\boldsymbol{\kappa}, \mathbf{r})
\end{aligned}
$$

which suggests that $\Phi(\boldsymbol{\kappa}, \mathbf{r})=\sum_{j} a\left(\boldsymbol{\kappa}+\mathbf{G}_{j}\right) e^{i\left(\boldsymbol{\kappa}+\mathbf{G}_{j}\right) \cdot \mathbf{r}}$, where $\mathbf{G}_{j}=j_{1} \boldsymbol{\beta}_{1}+j_{2} \boldsymbol{\beta}_{2}$ with $j_{1}, j_{2} \in \mathbb{Z}$, $\boldsymbol{\beta}_{1}=(N / \varrho, 0)$ and $\boldsymbol{\beta}_{2}=(-\tau N /(T \varrho), 2 \pi / T)$. From Eq.(12), we obtain the secular equation

$$
\operatorname{det}\left(\mathcal{H}_{l j}-E_{\kappa} \delta_{l j}\right)=0
$$


where $\mathcal{H}_{l j}=\frac{\hbar^{2}}{2 \mu}\left(\boldsymbol{\kappa}+\mathbf{G}_{j}\right)^{2} \delta_{l j}+\mathcal{U}_{l j}, \mathcal{U}_{l j}=\frac{1}{\Omega_{0}} \int_{\Omega_{0}} e^{i\left(\mathbf{G}_{l}-\mathbf{G}_{j}\right) \cdot \mathbf{r}} U_{s}(\mathbf{r}) d \mathbf{r}$ and $\Omega_{0}=\left|\boldsymbol{\alpha}_{1} \times \boldsymbol{\alpha}_{2}\right|$.

On the other hand, Periodic boundary condition along the circumference of the shell $S^{*}$ suggests that we just need to consider the first Brillouin zone which consists of $\boldsymbol{\kappa}=$ $\left(m_{v} / \varrho, \kappa_{z}\right)$ where $m_{v} \in \mathbb{Z}, 0 \leq m_{v}<N$ and $\kappa_{z} \in \mathbb{R}, 0 \leq \kappa_{z}<2 \pi / T$.

From Eq.(13), we can calculate the energy $E_{m_{v}, \kappa_{z}}$, and then the free energy $\mathcal{F}=$ $-\mathcal{N} \mathcal{T} \ln \mathcal{Z}, \frac{17}{1}$ where $\mathcal{Z}=\sum_{m_{v}} \int_{0}^{2 \pi / T} e^{-E_{m_{v}, \kappa_{z}} / \mathcal{T}} d \kappa_{z}$. Moreover, the specific heat per atom is 15

$$
c_{v}=-\frac{\mathcal{T} \partial^{2} \mathcal{F}}{\mathcal{N} \partial \mathcal{T}^{2}}=\frac{\left\langle E^{\prime 2}\right\rangle-\left\langle E^{\prime}\right\rangle^{2}}{\mathcal{T}^{2}},
$$

where $\left\langle E^{\prime}\right\rangle=\sum_{m_{v}=0}^{N-1} \int_{0}^{2 \pi / T} E_{m_{v}, \kappa_{z}} e^{-E_{m_{v}, \kappa_{z}} / \mathcal{T}} d \kappa_{z}$ and $\left\langle E^{\prime 2}\right\rangle=\sum_{m_{v}=0}^{N-1} \int_{0}^{2 \pi / T} E_{m_{v}, \kappa_{z}}^{2} e^{-E_{m_{v}, \kappa_{z}} / \mathcal{T}} d \kappa_{z}$.

In Fig 5 , the symbols " $\nabla$ " reflect $c_{v}$ varying with the temperature $\mathcal{T}$, which implies $\mathrm{Ne}$ atoms inside $(20,20)$ tube behave as the lattice gas 18 at ultra low temperature (specific heat is 0 ) and $2 \mathrm{D}$ gas at low temperature (specific heat approaches 1 ). There is no $1 \mathrm{D}$ gas inside $(20,20)$ tube, which is quite different from our usual notion.

Adopting the similar method, we calculate $c_{v}$ of Ne inside $(5,5),(10,10)$ and $(15,15)$ nanotubes and show them in Fig 4 and Fig 5 . When we obtain Fig 4, we have adopted the approximate potentials as $U(r)=4 \varepsilon\left[\left(\frac{\tilde{\sigma}}{\rho-r}\right)^{p_{1}}-\left(\frac{\tilde{\sigma}}{\rho-r}\right)^{p_{2}}\right]$. The parameters $\varepsilon, \tilde{\sigma}, \rho, p_{1}$, and $p_{2}$ are shown in Table I. These two figures imply that Ne inside four kinds of nanotubes exhibits 3D gas behavior at high temperature but different behaviors at low temperature: Ne inside $(5,5)$ nanotube behaves as $1 \mathrm{D}$ gas (specific heat is $1 / 2$ ) but inside $(10,10),(15$, $15)$, and $(20,20)$ nanotubes behaves as $2 \mathrm{D}$ gas. Furthermore, at ultra low temperature, Ne inside $(5,5)$ nanotube still displays 1D behavior but inside $(10,10),(15,15)$, and $(20,20)$ nanotubes behaves as lattice gas. Ne inside $(5,5)$ nanotube does not display $2 \mathrm{D}$ behavior and inside $(10,10),(15,15)$, and $(20,20)$ nanotubes does not display $1 \mathrm{D}$ behavior. Thus Ne inside nanotubes does not always display 1D behavior in spite of the remarkable aspect ratio of the length of tubes to their radius. Besides, Ne inside a definite nanotube may not display both 1D and 2D behaviors at different temperatures.

Our calculations support the view of dimensional crossover of gas inside SWNT's put forward by Cole et.al. ${ }^{7}$ but there is a significant difference: Cole et.al. obtain the dimensionality varying from one to two to three with the temperature increasing, but we obtain the dimensionality varying either from one directly to three or from two to three. The reason is that the potential of gas inside SWNT's selected by Cole et.al. departs father from the real 
case than that done by us.

If the potential on shell $S^{*}$ is uniform, one can intuitively expect the dimensionality to vary from one to two to three as the axial, azimuthal and radial degrees of freedom become excited as temperature increases. But the potential on this shell has more exquisite structure (the periodic structure) for large diameter nanotubes, such as $(10,10),(15,15)$ and $(20,20)$ tubes, which results in the approximate same axial and azimuthal excited energy. Therefore Ne in large diameter nanotubes exhibits lattice gas (0D), 2D and 3D behavior as temperature increases. On the contrary, the shell converges to the tube axis for small nanotube, e.g. $(5,5)$ tube, which results in the dimensionality varying from one direct to three as temperature increases.

The authors acknowledge the useful discussions with Prof. H. W. Peng, Dr. M. Li, F. Liu, Y. Zhang, L. R. Dai, and J. J. Zhou. Furthermore, we thank Dr. H. J. Zhou because he help us read the English.

* Email address tzc@itp.ac.cn

1 S. Iijima, Nature (London) 354, 56 (1991).

2 N. Hamada, S. I. Sawada, and A. Oshiyama, Phys. Rev. Lett. 68, 1579 (1992); R. Saito, M. Fujita, G. Dresselhaus, and M. S. Dresselhaus, Appl. Phys. Lett. 60, 2204 (1992); J. W. Mintmire, B. I. Dunlap, and C. T. White, Phys. Rev. Lett. 68, 631 (1992).

3 B. I. Yakobson and P. Avouris, Mechanical Properties of Carbon Nanotubes, in Carbon Nanotubes, edited by M. S. Dresselhaus and P. Avouris (Springer-Verlag, Berlin, 2001), pp. 287-327.

4 Z. C. Ou-Yang, Z. B. Su, and C. L. Wang, Phys. Rev. Lett. 78, 4055 (1997); X. Zhou, J. J. Zhou, and Z. C. Ou-Yang, Phys. Rev. B 62, 13692 (2000); Z. C. Tu and Z. C. Ou-Yang, Phys. Rev. B 65, 233407 (2002).

5 A. C. Dillon, K. M. Jones, T. A. Bekkedahl, C. H. Kiang, D. S. Bethune, and M. J. Heben, Nature 386, 377 (1997); C. Liu, Y. Y. Fan, M. Liu, H. T. Cong, H. M. Cheng, and M. S. Dresselhaus, Science 286, 1127 (1999).

6 W. Teizer, R. B. Hallock, E. Dujardin, and T. W. Ebbesen, Phys. Rev. Lett. 82, 5305 (1999); 84, 1844 (2000); A. Kuznetsova, J. T. Yates, Jr., J. Liu, and R. E. Smalley, J. Chem. Phys. 112, 9590 (2000); S. Talapatra, A. Zambano, S. E. Weber, and A. D. Migone, Phys. Rev. Lett. 
85, $138(2000)$.

7 M. Calbi, M. W. Cole, S. Gatica, M. J. Bojan and G. Stan, Rev. Mod. Phys. 73, 857 (2001); M. Calbi and M. W. Cole, Phys. Rev. B 66, 115413 (2002); S. Gatica, M. J. Bojan, G. Stan and M. W. Cole, J. Chem. Phys. 114, (2001); M. Calbi, F. Toigo and M. W. Cole, Phys. Rev. Lett. 86, 5062 (2001); G. Stan, M. W. Cole, J. Hartman, V. H. Crespi and S. Gatica, Phys. Rev. B 61, 7288 (2000); G. Stan, M. J. Bojan, S. Curtarola, S. Gatica and M. W. Cole, Phys. Rev. B 62, 2173 (2000); G. Stan, S. Gatica, M. Boninsegni, S. Curtarola and M. W. Cole, Am. J. Phys. 67, 1170 (1999); G. Stan and M. W. Cole, J. Low Temp. Phys. 110, 539 (1998); G. Stan, V. H. Crespi, M. W. Cole and M. Boninsegni, J. Low Temp. Phys. 113, 447 (1998).

8 C. Carraro, Phys. Rev. B 61, R16351 (2000); A. J. Zambano, S. Talapatra, and A. D. Migone, Phys. Rev. B 64, 75415 (2001); M. C. Gordillo, J. Boronat, and J. Casulleras, Phys. Rev. Lett. 85, $2348(2000)$.

9 J. C. Lasjaunias, K. Biljaković, J. L. Sauvajol, and P. Monceau, Phys. Rev. Lett. 91, 25901 (2003); J. C. Lasjaunias, K. Biljakovic; Z. Benes, J. E. Fischer, and P. Monceau, Phys. Rev. B 65, 113409 (2002).

10 R. Saito, M. S. Dresselhaus, and G. Dresselhaus, Physical Properties of Carbon Nanotubes (Imperial College Press, London, 1998).

11 J. O. Hirschfelder, C. F. Curtiss, and R. B. Bird, Molecular Theory of Gases and Liquids (John Wiley \& Sons, Inc., New York, 1954).

12 R. An (private communication); H. H. Peng and X. S. Xu, The Fundamentals of Theoretical Physics (Peking University Press, Beijing, 1998).

13 L. D. Landau and E. M. Lifshitz, Quantum Mechanics (Pergamon, Oxford, 1986).

14 Z. X. Wang and D. R. Guo, Introduction to Special Function (Peking University Press, Beijing, $2000)$.

15 L. D. Landau and E. M. Lifshitz, Statistical Physics (Pergamon, Oxford, 1986); L. E. Reichl, A Modern Course in Statistical Physics (John Wiley \& Sons, Inc., New York, 1998).

16 C. Kittel, Introduction to Solid State Physics (John Wiley \& Sons, Inc., New York, 1996).

17 In fact, here we just consider the energies in the first Brillouin zone. If considering $E_{\kappa}=E_{-\kappa}$ and $E_{\boldsymbol{\kappa}}=E_{\boldsymbol{\kappa}+\mathbf{G}_{j}}$, we need to multiple a constant before the partition function $\mathcal{Z}$ in the expression of the free energy.

18 M. M. Calbi, S. M. Gatica, M. J. Bojan, and M. W. Cole, cond-mat/0209220. 


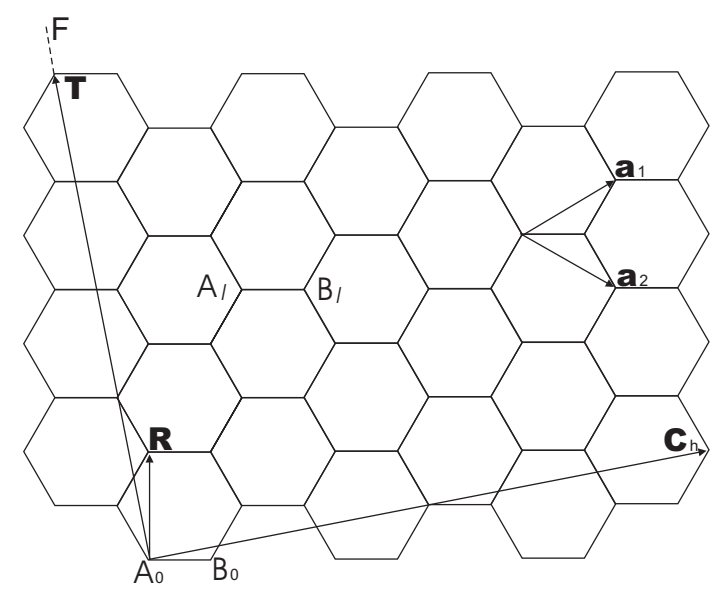

FIG. 1: The unrolled honeycomb lattice of a SWNT. By rolling up the sheet along the chiral vector $\mathbf{C}_{h}$, that is, such that the point $A_{0}$ coincides with the point corresponding to vector $\mathbf{C}_{h}$, a nanotube is formed. The vectors $\mathbf{a}_{1}$ and $\mathbf{a}_{2}$ are the real space unit vectors of the hexagonal lattice. The translational vector $\mathbf{T}$ is perpendicular to $\mathbf{C}_{h}$ and runs in the direction of the tube axis. The vector $\mathbf{R}$ is the symmetry vector. $A_{0}, B_{0}$ and $A_{l}, B_{l}(l=1,2, \cdots, N)$ are used to denote the sites of carbon atoms.

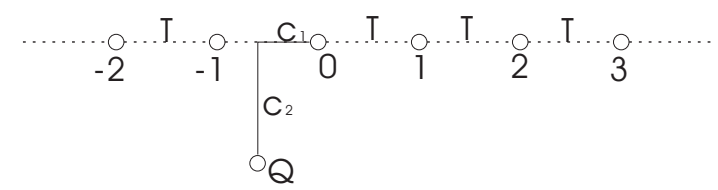

FIG. 2: An infinite atom chain and an atom Q out of the chain. Many atoms distribute regularly in a line form the infinite atom chain. The interval between neighbor atoms in the chain is $T$, and the site of atom $\mathrm{Q}$ relative to atom 0 can be represented by numbers $c_{1}$ and $c_{2}$.

TABLE I: The parameters in the approximate potentials for different nanotubes.

\begin{tabular}{cccccc}
\hline \hline$(\mathrm{n}, \mathrm{m})$ & $\varepsilon(\mathrm{K})$ & $\tilde{\sigma}(\AA)$ & $\rho(\AA)$ & $p_{1}$ & $p_{2}$ \\
\hline$(5,5)$ & 520 & 2.63 & 3.39 & 10 & 1 \\
$(10,10)$ & 360 & 2.63 & 6.78 & 10 & 3 \\
$(15,15)$ & 418 & 2.64 & 10.17 & 10 & 4 \\
\hline \hline
\end{tabular}




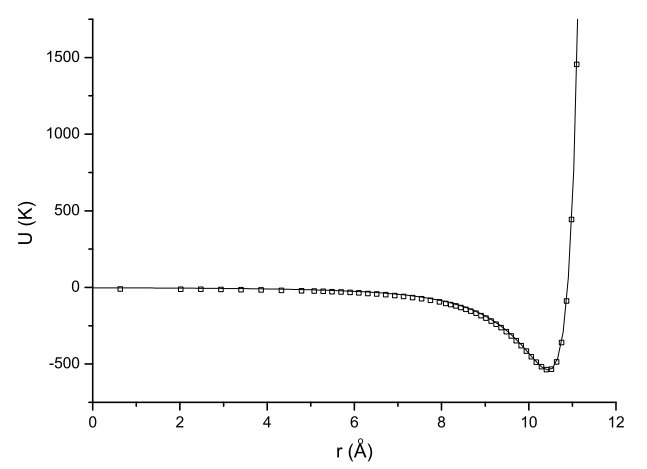

FIG. 3: The potentials inside the $(20,20)$ nanotube calculated from Eq.(15) (squires) and the fit curve (solid curve).

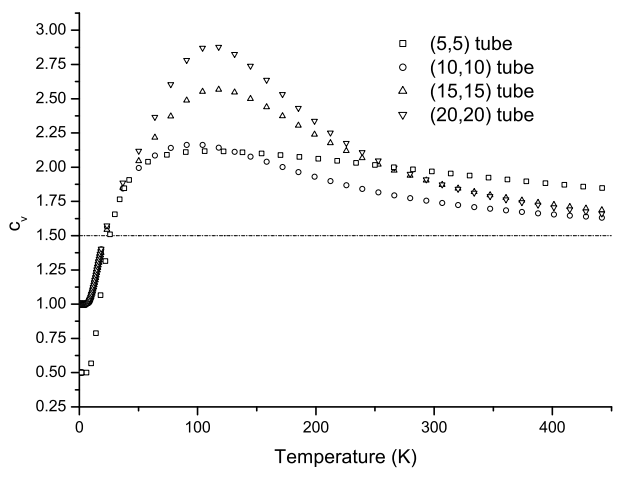

FIG. 4: The specific heats per atom $c_{v}$ of Ne inside $(5,5),(10,10),(15,15)$ and $(20,20)$ nanotubes under the approximate potentials varying with the temperature.

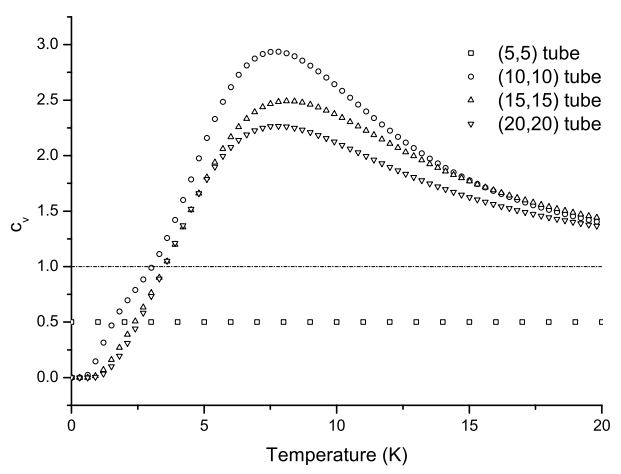

FIG. 5: The specific heat per atom $c_{v}$ of Ne inside $(5,5),(10,10),(15,15)$ and $(20,20)$ nanotubes at low temperatures. 